\title{
Long-term effectiveness of levodopa-carbidopa intestinal gel on motor and non-motor symptoms in advanced Parkinson's disease: results of the Italian GLORIA patient population
}

\author{
Angelo Antonini ${ }^{1}$ - Pietro Marano ${ }^{2} \cdot$ Graziano Gusmaroli $^{3} \cdot$ Nicola Modugno $^{4}$. Claudio Pacchetti ${ }^{5}$. \\ Mariachiara Sensi ${ }^{6} \cdot$ Gabriella Melzi $^{7} \cdot$ Lars Bergmann $^{8} \cdot$ Maurizio Zibetti $^{9} \cdot$ Leonardo Lopiano $^{9}$
}

Received: 20 March 2019 / Accepted: 7 April 2020 / Published online: 28 April 2020

(C) The Author(s) 2020

\begin{abstract}
Introduction The GLORIA registry included 375 advanced Parkinson's disease (PD) patients and evaluated the efficacy and safety of a 24-month levodopa-carbidopa intestinal gel (LCIG) treatment in routine medical care. This analysis focuses on the Italian population, 60 patients treated with LCIG in 7 specialised PD care centres.

Methods Hours of "Off" and "On" time were assessed with a modified version of the Unified Parkinson's Disease Rating Scale (UPDRS) part IV items 39 and 32. Motor fluctuations, dyskinesia, non-motor symptoms, quality of life and safety were evaluated. Results Overall, 42 (70\%) out of 60 patients completed the registry. LCIG treatment reduced "Off" time $(-3.3 \pm 2.7 \mathrm{~h}$ at month 24 (M24), $P<0.0001)$, increased "On" time with dyskinesia $(-2.6 \pm 5.2 \mathrm{~h}$ at M12, $P=0.0160)$, and improved UPDRS II and UPDRS III total scores at M24 ( $-4.5 \pm 10.6, P=0.0333$ and $-4.9 \pm 11.7, P=0.0229$, respectively), Non-Motor Symptom Scale (NMSS) total score $(-21.8 \pm 28.5, P<0.0001)$ and Parkinson's Disease Questionnaire-8 item (PDQ- 8$)$ total score $(-12.5 \pm 23.9$, $P=0.0173$ ) versus previous oral therapy. Adverse drug reactions (ADR) possibly or probably related to treatment were reported in $16(28.6 \%)$ patients. Decreased weight (7.1\%), polyneuropathy (7.1\%) and abdominal pain (5.4\%) were the most frequent ADRs while device malfunction (5.4\%) and medical device change (5.4\%) were the most reported device complaints.

Conclusions LCIG improved motor fluctuations, non-motor symptoms and quality of life over 24 months while tolerability was consistent with the established safety profile.
\end{abstract}

Keywords Parkinson's disease $\cdot$ Levodopa-carbidopa intestinal gel $\cdot$ Motor symptoms $\cdot$ Non-motor symptoms · Quality of life · Routine patient care

Angelo Antonini

angelo3000@yahoo.com

1 Parkinson and Movement Disorders Unit, Department of Neuroscience, University of Padua, Padua, Italy

2 Madonna del Rosario Clinic, Catania, Italy

3 Neurology Unit, Ospedale degli Infermi, Biella, Italy

4 IRCCS Neuromed, Pozzilli, Italy

5 Parkinson's Disease and Movement Disorders Unit, IRCCS C. Mondino Foundation, Pavia, Italy

6 Neurology Unit, Sant'Anna University Hospital, Ferrara, Italy

7 Medical Department, AbbVie s.r.l., Campoverde di Aprilia, Italy

8 AbbVie Inc., North Chicago, IL, USA

9 Department of Neuroscience, University of Torino, AOU Città della Salute e della Scienza, Torino, Italy

\section{Introduction}

Parkinson's disease (PD) is a progressive neurodegenerative disorder, and levodopa is affirmed as the most effective drug for treatment [1]. PD in advanced stage and long-term oral levodopa administration may account for disabling motor complications [2,3]. Although primarily considered as a movement disorder, $\mathrm{PD}$ patients present a broad range of non-motor symptoms (NMS) [4, 5], significantly contributing to co-morbidities and loss of autonomy, leading to a decline of quality of life (QoL) [6] and increase in PD-related health care costs [7, 8].

As PD progresses, the duration of the levodopa response shortens and the therapeutic window narrows. Fluctuating peripheral levodopa plasma levels, caused, e.g. by erratic gastric emptying, increasingly provoke motor fluctuations [9-11] and severely interfere with daily activities, social interactions and patient's quality of life (QoL) $[12,13]$. 
Levodopa-carbidopa intestinal gel (LCIG) is continuously released to the upper intestine and helps to achieve more stable levodopa plasma levels compared to oral levodopa administration $[14,15]$. As a consequence, motor fluctuations decrease and non-motor symptoms [16-20] and QoL improve [12, 13].

To date, only a few studies evaluated the long-term efficacy and safety of LCIG treatment in routine clinical care in a large cohort of advanced PD patients [8, 18, 21-24]. The GLORIA registry systematically assessed the long-term effectiveness of LCIG on motor symptoms as well as on non-motor symptoms (NMS) and QoL; in addition, safety of LCIG was evaluated.

Due to the observational nature of the GLORIA registry reflecting the routine clinical care for LCIG management, the aim of this paper was to assess the outcomes of the Italian patient cohort treated in 7 specialised PD care centres and to detect differences with the results of the overall $\mathrm{PD}$ population included in GLORIA study across 18 countries $[25,26]$.

\section{Methods}

\section{Study design}

Seventy-five specialised PD care unit centres across 18 countries included 375 patients with advanced PD experiencing motor complications in this 24-month multi-national, non-interventional, observational registry. According to the standard medicinal product characteristics, the PD patient and the treating neurologist should jointly decide to switch to LCIG treatment when oral and transdermal treatments cannot be further optimised. Clinical outcomes were recorded every 6 months for the initial 24-month LCIG treatment. The study protocol was approved by health authorities and national and/or local independent ethics committees in each country and at each participating centre.

The outcomes of the 12-month interim and the final 24month analysis of the GLORIA registry were published [25, 26]. This paper presents the results of the Italian study population, 60 patients treated with LCIG at the 7 Italian PD care unit centres.

For statistical analyses, ANOVA over time and paired $t$ tests were performed for the comparison of all efficacy outcomes to baseline (BL).

\section{Patients}

All patients provided written informed consent prior to inclusion. In LCIG-naïve patients (63\%), all observations were recorded prospectively. Some of the patients received LCIG for $\leq 12$ months before inclusion, and clinical data were collected retrospectively up to enrolment and were recorded thereafter prospectively as of the M12 to the M24 follow-up. LCIG treatment was initiated in the majority of patients by using a temporary nasojejunal (NJ) tube to titrate and optimise the dose before being administered through percutaneous endoscopic gastrostomy with jejunal extension (PEG-J) (according to local label and reimbursement criteria).

\section{Efficacy}

The actual hours of "Off" time and "On" time with dyskinesias were assessed using the Unified Parkinson's Disease Rating Scale IV (UPDRS) Items 39 and 32 modified according to the Movement Disorder Society (MDS)-UPDRS (corresponding parts 4.3 and 4.1). The UPDRS parts II, III, and IV were assessed in the "On" state. Non-motor symptoms were evaluated using the NMSS and patient-reported quality of life (QoL) measures included the disease-specific PDQ-8. Efficacy assessments were collected at baseline (BL) before LCIG treatment initiation, at discharge from hospital following PEG-J placement (day 1-D1), at month 6 (M6), M12, M18, and M24. All outcomes at follow-up visits were analysed as mean change from baseline.

\section{Safety}

Adverse drug reactions (ADRs), considered by the investigator as adverse events with a reasonable possible causal relationship to the treatment drug or the device, were recorded and coded according to the Medical Dictionary for Regulatory Activities (MedDRA) [27] and categorised by the study investigator as mild, moderate, or severe, with unlikely, possible of probable relationship to the drug/device system. Serious ADRs and product complaints were monitored and recorded.

\section{Results}

\section{Patient population, demographics, and disease characteristics at baseline}

Out of the 60 patients enrolled, 42 (70\%) completed the registry. Patient demographics, PD characteristics, and baseline assessments of motor symptoms, NMSS, and QoL are summarised in Table 1. Reasons for premature discontinuation $(N=15,25 \%)$ were withdrawal of consent ( $N=7,4$ withdrawals between BL and D1 visit), lack of efficacy $(N=3)$, adverse events (ADRs, concomitant diseases, or death; $N=2)$, protocol violations $(N=2)$, and administrative reasons $(N=1)$. No return to follow-up visits was reported for $3(5 \%)$ patients.

\section{Treatments}

The most common concomitant medications across all study visits were oral levodopa and dopamine agonists 
and catechol-O-methyltransferase (COMT) inhibitors (Table 1). Among patients who received LCIG as a monotherapy, the mean daily levodopa equivalent dose (LED) [28] ranged from $1278 \pm 675 \mathrm{mg}$ at D1 $(n=19)$ to a maximum of $1456 \pm 529 \mathrm{mg}$ at M12 $(n=15)$ and to $1396 \pm$ $585 \mathrm{mg}$ at M24. The mean daily LED for patients with LCIG combination therapy ranged from $1814 \pm 744 \mathrm{mg}$ at $\mathrm{D} 1(n=36)$ to a maximum of $1847 \pm 744 \mathrm{mg}$ at M6 $(n=$ $30)$ and to $1845 \pm 713 \mathrm{mg}$ at M24.

\section{Efficacy}

A significant reduction from $\mathrm{BL}$ for the mean $( \pm \mathrm{SD})$ number of "Off" hours was observed through the entire study period. Results at D1 $(-1.4 \pm 2.5 \mathrm{~h} ; P=0.0070)$ and M24 $(-3.3 \pm$ $2.7 \mathrm{~h} ; P<.0001)$ versus BL are reported. Moreover, compared to BL, the mean "On" time with dyskinesia showed a significant improvement at D1 $(-1.6 \pm 3.9 \mathrm{~h} ; P=0.0491), \mathrm{M} 6$ and M12 ( $2.6 \pm 5.1 \mathrm{~h} ; P=0.0160)$ (Fig. 1a and b). UPDRS II and III scores (assessed when "On") showed significant reductions compared to BL through M24 (-4.5 $\pm 10.6 ; P=$ 0.0333 and $-4.9 \pm 11.7 ; P=0.0229$ ), respectively (Fig. 2a and $b$ ). The NMSS total score was significantly reduced compared to $\mathrm{BL}$ at all study visits with a maximum mean change of $-25.4 \pm 33.6$ at M18 $(P=0.0009)$ (Fig. 3a). The PDQ-8 total score was significantly reduced at every study visit ($12.5 \pm 23.9$ at M24; $P=0.0173$ ) (Fig. 3b).

\section{Safety}

Overall, 16 (28.6\%) patients experienced one or more ADRs (Table 2). The most frequently reported ADRs were decreased weight $(7.1 \%)$, polyneuropathy $(7.1 \%)$ and abdominal pain (5.4\%), while device malfunction (5.4\%) and medical device change $(5.4 \%)$ were the most frequent device complaints. These ADRs were generally transient. Serious and severe ADRs were reported in $17.9 \%$ and $10.7 \%$ patients, respectively, and one patient (1.8\%) with an ADR leading to LCIG discontinuation was reported. Two deaths occurred during the registry period, one for myocardial infarction and one for pulmonary oedema, both deemed as unlikely related by the investigator's judgement.

\section{Discussion}

The GLORIA registry is one of the largest cohorts of advanced PD patients treated with LCIG and provided real-life evidence for the long-term effectiveness in reducing motor fluctuations and dyskinesia during routine clinical care. In addition, marked improvements in non-motor symptoms and QoL were demonstrated over the 24-month treatment period.
Table 1 Demographics, medical history, disease characteristics and previous $\mathrm{PD}$ treatments recorded at baseline (BL) presented in mean \pm $\mathrm{SD}$ or number $(\%)$

\begin{tabular}{|c|c|c|}
\hline \multirow[t]{2}{*}{ Gender $^{\mathrm{a}}$} & Male & $37(61.7)$ \\
\hline & Female & $23(38.3)$ \\
\hline \multicolumn{2}{|c|}{ Age (years) ${ }^{a}$} & $68.3 \pm 8.1$ \\
\hline \multicolumn{2}{|c|}{ Time since PD diagnosis (years) ${ }^{\mathrm{a}}$} & $11.7 \pm 5.8$ \\
\hline \multicolumn{3}{|c|}{ Hoehn and Yahr ${ }^{\mathrm{b}}$} \\
\hline \multicolumn{2}{|c|}{ During “On" } & $2.7 \pm 0.6$ \\
\hline \multicolumn{2}{|c|}{ During "OFF" } & $3.6 \pm 0.8$ \\
\hline \multicolumn{3}{|c|}{ UPDRS Part IV ${ }^{\mathrm{b}}$} \\
\hline \multicolumn{2}{|c|}{ Modified item 39: “Off” phase (h/day) } & $4.3 \pm 2.7$ \\
\hline \multicolumn{2}{|c|}{ Modified item 32: dyskinesia (h/day) } & $4.7 \pm 4.1$ \\
\hline \multicolumn{2}{|c|}{ UPDRS Part II (activities of daily living) "On" } & $18.9 \pm 10.4$ \\
\hline \multicolumn{2}{|c|}{ UPDRS Part III (motor examination) ${ }^{\mathrm{b}}$ “On” } & $29.4 \pm 10.8$ \\
\hline \multicolumn{2}{|c|}{ NMSS total score ${ }^{\mathrm{b}}$} & $66.0 \pm 45.1$ \\
\hline \multicolumn{2}{|c|}{ PDQ-8 total score ${ }^{\mathrm{b}}$} & $52.6 \pm 21.7$ \\
\hline \multicolumn{2}{|c|}{ EQ-5D score ${ }^{\mathrm{b}}$} & $0.33 \pm 0.35$ \\
\hline \multicolumn{2}{|c|}{ EQ-VAS score ${ }^{\mathrm{b}}$} & $45.8 \pm 23.4$ \\
\hline \multicolumn{3}{|c|}{ PD medications reported at BL } \\
\hline \multicolumn{2}{|l|}{ Levodopa } & $57(95.0)$ \\
\hline \multicolumn{2}{|c|}{ Total daily oral dose (mg) } & $846 \pm 361$ \\
\hline \multicolumn{2}{|c|}{ Dopamine agonist } & $44(73.3)$ \\
\hline \multicolumn{2}{|c|}{ COMT inhibitors } & $31(51.7)$ \\
\hline \multicolumn{2}{|c|}{ MAO-B inhibitors } & $12(20.0)$ \\
\hline \multicolumn{2}{|c|}{ Amantadine } & $11(18.3)$ \\
\hline \multicolumn{2}{|l|}{ Other oral } & $9(15.0)$ \\
\hline
\end{tabular}

PD Parkinson's disease, UPDRS Unified Parkinson's Disease Rating Scale, NMSS non-motor symptom scale, $P D Q-8$ Parkinson's Disease Questionnaire 8-item, EQ-5D Euro Quality of Life 5 Dimensions, $E Q$ VAS Euro Quality of Life Visual Analog Scale, COMT catechol-O-methyltransferase, $M A O-B$ monoamine oxidase-B

${ }^{\mathrm{a}}$ All subjects consented population $(N=60)$

${ }^{\mathrm{b}}$ Full analysis set population $(N=52)$

The analysis of the Italian sub-population consisting of 60 patients included by 7 specialised PD care centres in Italy revealed a significant reduction of the time patients spent in the "Off" condition. Patients treated with LCIG experienced a $57 \%, 87 \%$ and $75 \%$ reduction from BL in "Off" time at day 1, M18 and M24, respectively. This reduction in "Off" time was well above what is deemed clinically relevant [29] and was consistent with or even better than published open-label studies and randomised controlled trials on LCIG $[8,16-26]$ and higher compared to a $65 \%$ reduction in "Off" time at M24 demonstrated in the GLORIA registry [26]. In fact, in this sub-population analysis, we found a $77 \%$ reduction of the mean daily "Off" time compared to baseline.

The "On" time with dyskinesia was significantly reduced from BL in patients treated with LCIG by $34 \%$ and 
Fig. 1 Motor symptom efficacy: mean change from BL of daily hours of a "Off" time (modified UPDRS Part IV Items 39) and b

"On" time with dyskinesia (modified UPDRS Part IV Item 32) at start of LCIG treatment with permanent tube (D1), at M6, M12, M18 and M24 compared to BL in a paired $t$ test at the $P<0.05$ (*), $P<0.01(* *)$ and $P<0.001$ $(* * *)$. Numbers indicated in brackets represent the numbers of matched pairs. Error bars indicate SD. UPDRS $=$ Unified

Parkinson's Disease Rating Scale; $\mathrm{BL}=$ baseline; $\mathrm{D} 1=$ discharge from hospital post-PEG-J placement; $\mathrm{M}=$ month a

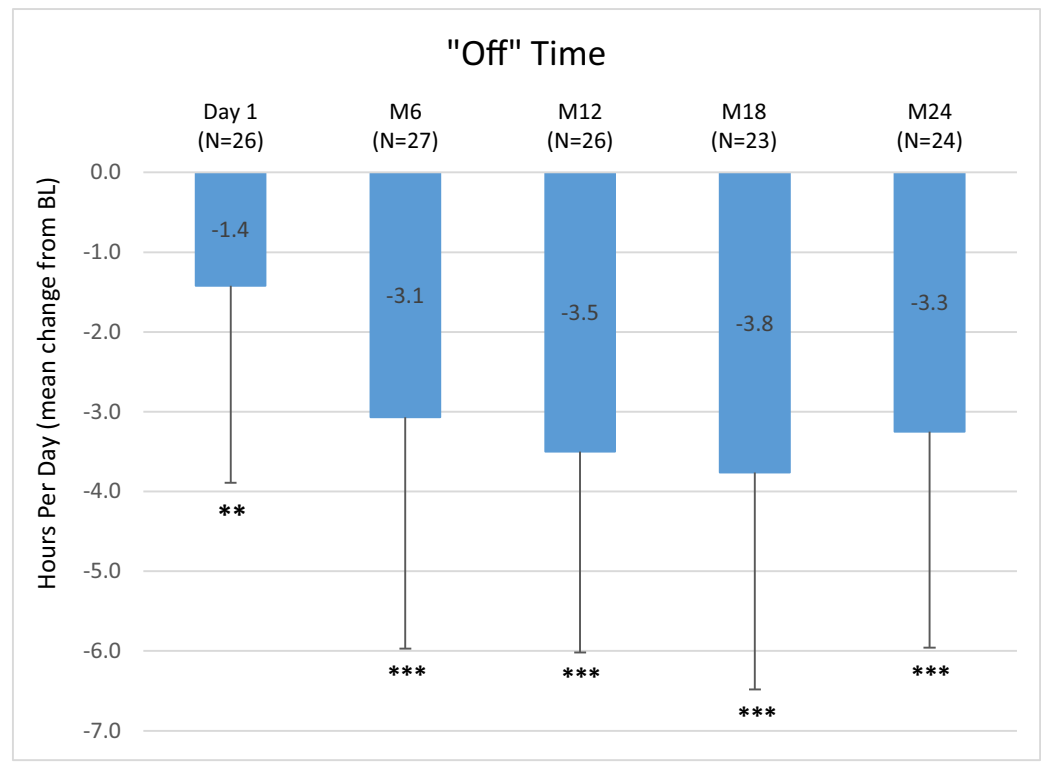

b

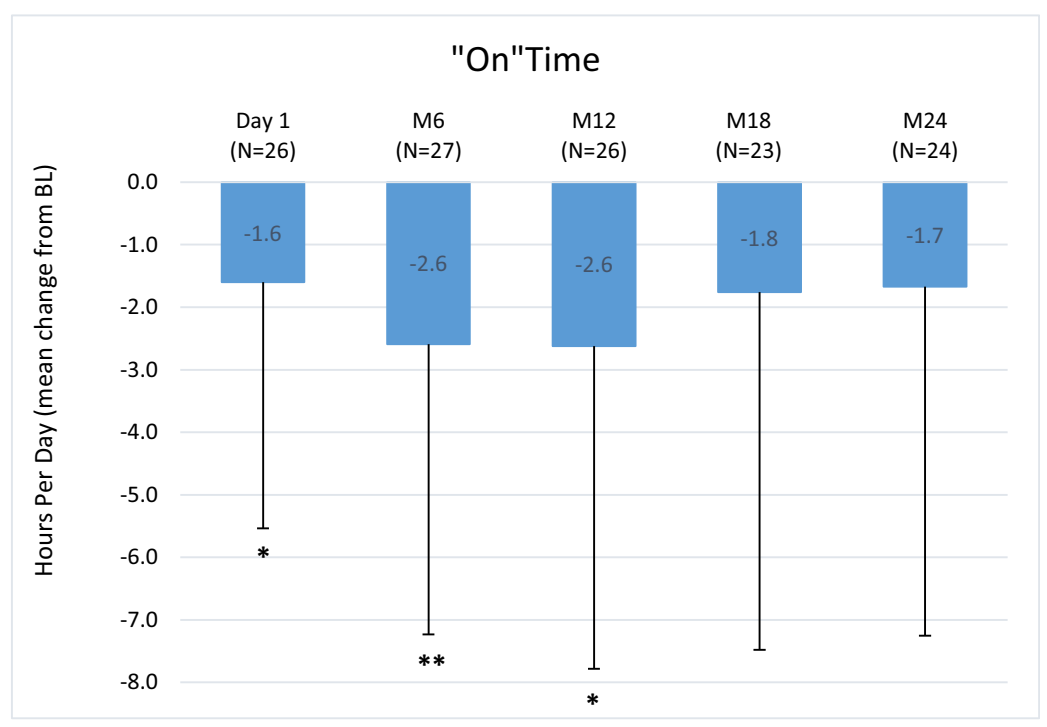

$56 \%$ at D1 and M12, respectively. The magnitude of reduction was similar at M18 and M24, however not significant, most likely due to the small number of observations. These outcomes reflect the current opinion that switching to continuous levodopa delivery not only improves motor fluctuations, but also reduces pre-existing dyskinesia. In the GLORIA population, the average "On" time with dyskinesia was reduced by $25 \%$ at all visits [26]. The marked reduction in "OFF" time and parallel substantial decrease in "On" time with dyskinesia at day 1 were achieved by switching from oral PD medications to LCIG. Importantly, these improvements in "OFF" time and "On" time with dyskinesia were obtained maintaining a stable LED throughout the 24-month LCIG treatment, reflecting experience in using LCIG and good treatment follow-up of patients. In contrast, the LED increased slightly in the total GLORIA population [26]. In this registry, no specific recommendations were provided for optimisation of oral doses prior to switching to LCIG and titration of LCIG during the initiation of infusion. The applied individual procedures reflected the 'real-world' routine clinical care. Treatment with LCIG led to a significant $20 \%$ to $38 \%$ reduction in NMSS score throughout the study which together with motor improvement resulted in a significant $14 \%$ to $30 \%$ reduction of the PDQ- 8 score throughout the 24 month treatment $[25,26]$. Also, the percentage reductions 
Fig. 2 Motor symptoms a of UPDRS II and $\mathbf{b}$ UPDRS III scores at start of LCIG treatment with permanent tube (D1), at M6, M12, M18 and M24 compared to BL in a paired $t$ test at the $P<0.05$ (*), $P<0.01$ (**) and $P<0.001$ $(* * *)$. Numbers indicated in brackets represent the numbers of matched pairs. Error bars indicate SD. UPDRS $=$ Unified Parkinson's Disease Rating Scale; Part II (activities of daily living) and Part III (motor examination); $\mathrm{BL}=$ baseline; $\mathrm{D} 1$ = discharge from hospital post-PEG-J placement; $\mathrm{M}=$ month a

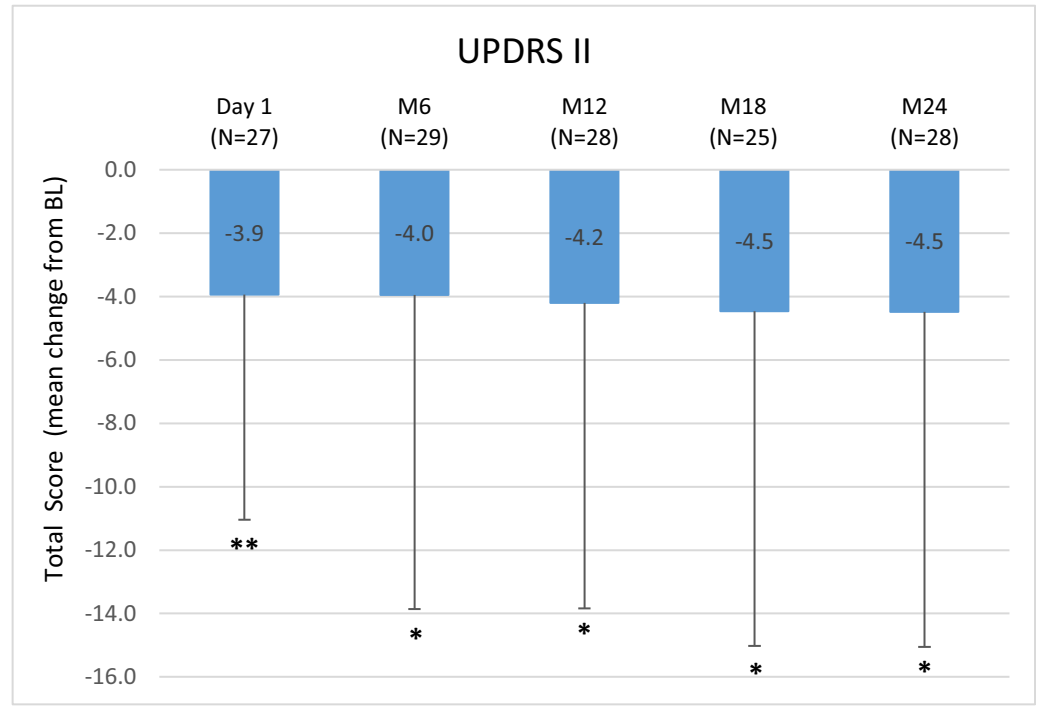

b

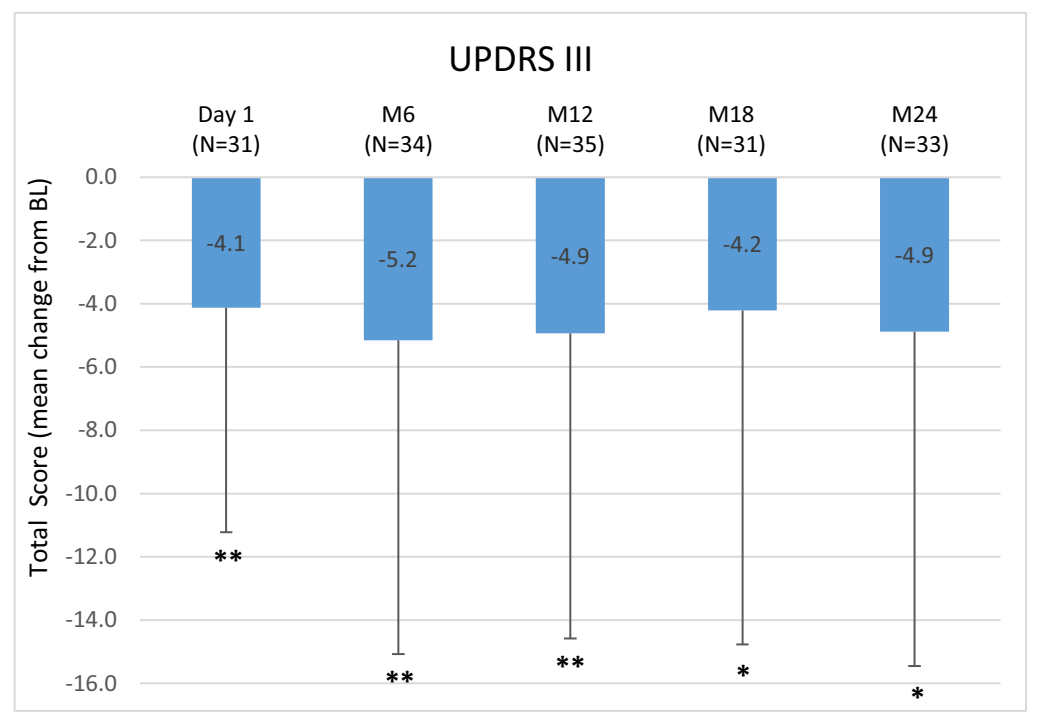

of NMS and PDQ-8 vs BL were greater in the Italian subgroup analysis compared to the total GLORIA population even if the Italian group baseline "Off" time was lower $(4.3 \pm 2.7$ vs $6.0 \pm 3.2 \mathrm{~h})$. This observation seems of interest considering that in another GLORIA sub-analysis, it was recently reported that the magnitude of QoL improvement was greater in patients with more "Off" time and larger LED dose at baseline [30]. LCIG led to significant improvements in UPDRS II and III scores, 24\% and 17\%, respectively, at M24. These data are similar to those reported in previous studies examining LCIG treatment $[2,6,8$, $9,23]$ and better to the one reported in the GLORIA population [26].

Since only ADRs, considered by the investigator as adverse AEs with a reasonable causal relationship to the treatment (drug/device), were recorded in GLORIA with less intense monitoring compared to clinical studies, the safety analyses of this registry cannot be directly compared with the results of clinical studies. Furthermore, the safety outcomes of the Italian cohort compared to the GLORIA population were different, most likely due to local reporting procedure and other factors. In the Italian cohort, only $29 \%$ of the patients reported ADRs, while in the GLORIA population, $55 \%$ of the patients reported ADRs. Device and procedure-related events were the most frequently reported ADRs in the GLORIA population, yet were less frequently reported in the Italian cohort. These ADRs were generally transient, occurring mainly within the first month post-PEG-J placement. This outcome emphasises the importance for a close 
Fig. 3 a Non-motor symptom improvements (NMSS total score reduction) and $\mathbf{b}$ quality of life improvement (PDQ-8 score reduction) at start of LCIG treatment with permanent tube (D1), at M6, M12, M18 and M24 compared to BL in a paired $t$ test at the $P<0.05$ (*), $P<0.01$ (**) and $P<0.001$ (***). Numbers indicated in brackets represent the numbers of matched pairs. Error bars indicate SD. NMSS = nonmotor symptom scale, PDQ-8 = Parkinson's Disease

Questionnaire 8-item; BL = baseline; $\mathrm{D} 1=$ discharge from hospital post-PEG-J placement; $\mathrm{M}=$ month a

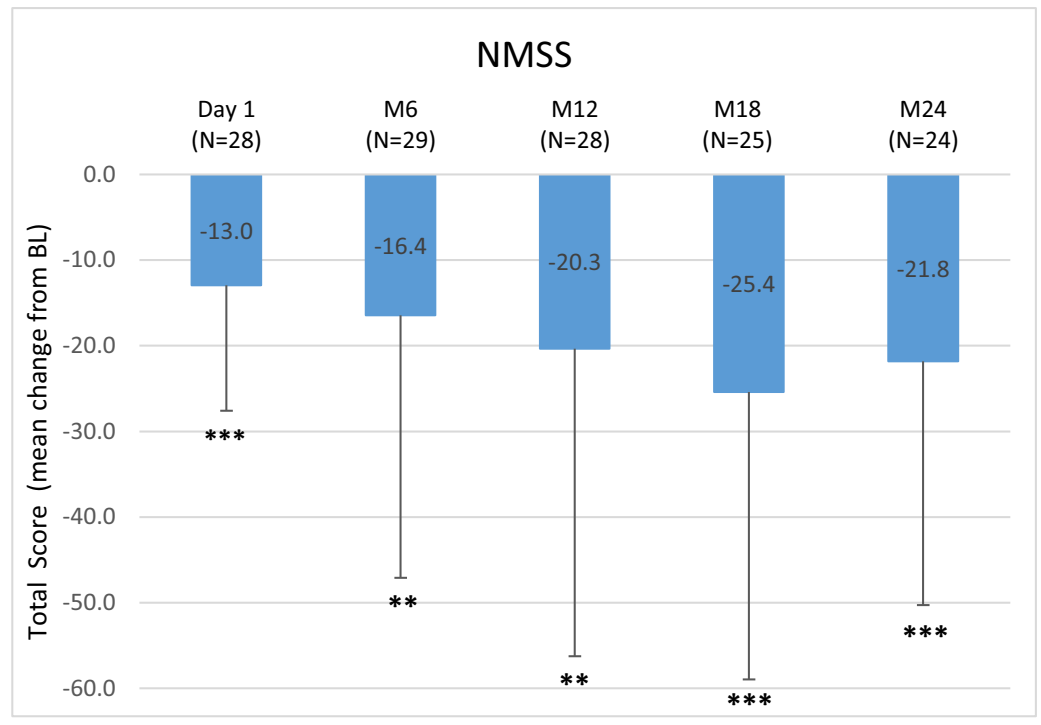

b

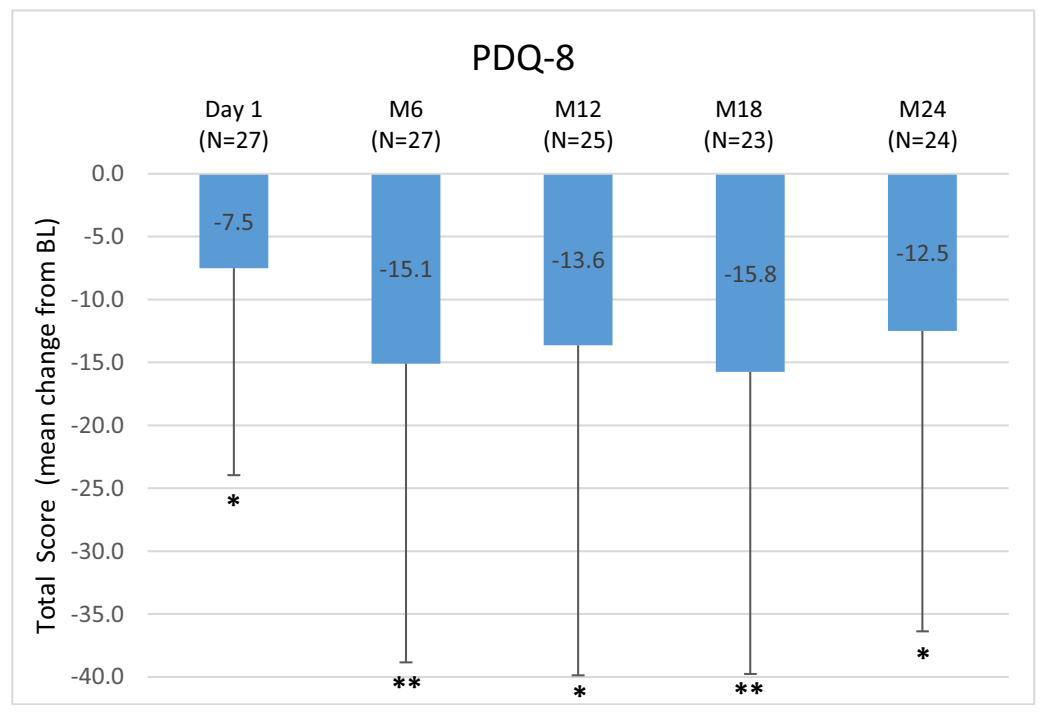

collaboration between the neurologist and gastroenterologist during the PEG-J placement and the need for an intense monitoring in the immediate post-PEG-J placement period. Also, this may reflect the long-standing experience of the seven specialised Italian PD care centres. Overall, the most frequently reported ADRs and the GIrelated ADRs were consistent with the known long-term complications of the PEG-J procedure [31] and the established safety profile of LCIG [16-18, 32].

Considering the mean age (68 years) and disease characteristics of the Italian patient population, LCIG procedures and treatment were generally well tolerated, with a lower rate of discontinuation due to ADR (1.8\%) compared to a reported rate for the GLORIA population (7\%) [26]. Out of the two deaths occurring during the registry, one was not related to treatment (myocardial infarction) and one unlikely related (pulmonary oedema).
This analysis provides an important "real life" insight in the LCIG treatment of advanced PD patients and revealed some specific facets on LCIG experience and treatment modalities in Italy. However, the design of this registry includes some limitations, such as the registry's open-label design, the lack of a control group, not allowing to compare efficacy and safety assessments, and a partially retrospectively collection of data in some of the patients up to M12, while in all naïve LCIG patients $(63 \%)$, all data were collected prospectively. However, according to the pre-defined sub analysis of retroversus prospective data collection, there were no differences in the total population. In addition, a potential variability of data could be due to the broad geographical distribution of the participating sites in 18 countries, with the potential for different treatment approaches $[25,26]$. The latter was addressed with this analysis of the Italian population comparing the results with the overall GLORIA outcomes. 
Table 2 Tolerability of LCIG infusion: overall summary of ADRs/ product complaints reported during LCIG infusion with temporary NJ tube and permanent PEG/J, ADRs reported with an incidence of $\geq 3 \%$ and all serious ADRs (\% of $N=56$ )

\begin{tabular}{ll} 
Patients with at least one ADR* & $16(28.6)$ \\
Patients with at least one possibly or probably related* & $13(23.2)$ \\
Patients with at least one serious* $^{*}$ & $10(17.9)$ \\
Patients with at least one severe* $^{*}$ & $6(10.7)$ \\
Patients with at least one leading to LCIG discontinuation* & $1(1.8)$ \\
ADRs or product complaints occurring in $\geq 3 \%$ of patients $^{\mathrm{a}}$ & \\
Weight decreased & $4(7.1)$ \\
Polyneuropathy & $4(7.1)$ \\
Abdominal pain & $3(5.4)$ \\
Device malfunction & $3(5.4)$ \\
Medical device change & $3(5.4)$ \\
Gastrointestinal stoma complication & $2(3.6)$ \\
Delirium & $2(3.6)$ \\
Serious ADRs or product complaints occurring in $\geq 1 \%$ of patients & \\
Pneumoperitoneum & $2(3.6)$ \\
Delirium & $2(3.6)$ \\
Abdominal pain & $1(1.8)$ \\
Ileus & $1(1.8)$ \\
Dyskinesia & $1(1.8)$ \\
Polyneuropathy & $1(1.8)$ \\
Somnolence & $1(1.8)$ \\
Anaemia & $1(1.8)$ \\
Myocardial infarction & $1(1.8)$ \\
Device malfunction & $1(1.8)$ \\
Pneumonia & $1(1.8)$ \\
& $1(1.8)$ \\
\hline
\end{tabular}

Note: “*” Denominator is count of patients in the regarding population

Gastrointestinal and gastrointestinal procedure-related ADRs are italicised

${ }^{a}$ Data indicates incidence of ADRs

${ }^{\mathrm{b}}$ During 24 months of LCIG infusion via PEG-J

$A D R$ adverse drug reaction (adverse events with a possible/probable relationship to the treatment drug or device), GI gastrointestinal, $L C I G$ levodopa-carbidopa intestinal gel, $P E G-J$ percutaneous endoscopic gastrostomy with jejunal extension

In conclusion, LCIG resulted in sustained reductions over the 24-month treatment period in motor fluctuations and NMS burden and improvement of QoL, in advanced PD patients in routine care in Italian PD care centres. The tolerability of LCIG was consistent with the previously established safety profile.

Acknowledgements The EDC system used to collect clinical data was provided by Köhler eClinical Ltd., Freiburg, Germany. The statistical analysis was performed by Christoph Meyenberg, Köhler eClinical Ltd., Freiburg, Germany, funded by AbbVie. The authors would like to acknowledge the following non-author, GLORIA study investigators for their contributions: Maria Rosaria Seminara, Catania, Italy and Francesca Preda, Ospedale Bufalini, Cesena, Italy.

Authors' contributions and disclosures Angelo Antonini was a study investigator received honorarium from $\mathrm{AbbVie}$ and has received compensation for consultancy and speaker-related activities from AbbVie, UCB, Lundbeck, Bial, Neuroderm, Boehringer Ingelheim, Mundipharma and Zambon. Dr. Antonini's, contributions include chairperson of the advisory board, study concept and design, acquisition and interpretation of the data, and review and critique of the manuscript.

Pietro Marano was a study investigator and has received honorarium from AbbVie. Dr. Marano contributions include acquisition and interpretation of the data, and review and critique of the manuscript.

Graziano Gusmaroli was a study investigator and has received honorarium from AbbVie. Dr. Gusmaroli contributions include acquisition and interpretation of the data, and review and critique of the manuscript.

Nicola Modugno was a study investigator and has received compensation for speaker-related activities from AbbVie and UCB Pharma. Dr. Modugno contributions include acquisition and interpretation of the data, and review and critique of the manuscript.

Claudio Pacchetti was a study investigator and has received honorarium from AbbVie; Boston Scientific, Italy; Medtronic, Italy; Abbott, Italy. Dr. Pacchetti contributions include acquisition and interpretation of the data, and review and critique of the manuscript.

Mariachiara Sensi was a study investigator and has received compensation for speaker-related activities from AbbVie. Dr. Sensi contributions include acquisition and interpretation of the data, and review and critique of the manuscript.

Gabriella Melzi is an employee of AbbVie s.r.l., Italy. Gabriella Melzi's contributions include acquisition and interpretation of the data, and review and critique of the manuscript.

Lars Bergmann is an employee of AbbVie and holds AbbVie stock and/or stock options. Dr. Bergmann's contributions include acquisition and interpretation of the data, and review and critique of the manuscript.

Maurizio Zibetti was a study investigator and has received honorarium from Medtronic, Zambon, UCB Pharma and AbbVie. Dr. Zibetti contributions include acquisition and interpretation of the data, and review and critique of the manuscript.

Leonardo Lopiano has received honoraria for consulting services and symposia from AbbVie. Dr. Lopiano contributions include acquisition and interpretation of the data, and review and critique of the manuscript.

Funding information This study was sponsored by AbbVie Inc. (North Chicago, IL, USA), which participated in the study design, research, data collection, analysis. Interpretation of the Italian subpopulation data, writing, reviewing and approving the publication has been funded and supported by the Medical Department of AbbVie S.r.l., Italy.

Medical writing support was provided by Urs E. Gasser, ClinResearch Ltd., Switzerland, funded by AbbVie.

\section{Compliance with ethical standards}

\section{Conflict of interest None.}

Ethical approval The study protocol was approved by the local ethics committees in each participating centre in Italy.

Open Access This article is licensed under a Creative Commons Attribution 4.0 International License, which permits use, sharing, adaptation, distribution and reproduction in any medium or format, as long as you give appropriate credit to the original author(s) and the source, provide a link to the Creative Commons licence, and indicate if changes were made. The images or other third party material in this article are included in the article's Creative Commons licence, unless indicated otherwise in a 
credit line to the material. If material is not included in the article's Creative Commons licence and your intended use is not permitted by statutory regulation or exceeds the permitted use, you will need to obtain permission directly from the copyright holder. To view a copy of this licence, visit http://creativecommons.org/licenses/by/4.0/.

\section{References}

1. Tambasco N, Romoli M, Calabresi P (2018) Levodopa in Parkinson's Disease: Current status and future developments. Curr Neuropharmacol 16:1239-1252

2. Obeso JA, Olanow CW, Nutt JG (2000) Levodopa motor complications in Parkinson's disease. Trends Neurosci 23:S2

3. Fabbrini G, Brotchie JM, Grandas F, Nomoto M, Goetz CG (2007) Levodopa induced dyskinesias. Mov Disord 22:1379-1389

4. Antonini A, Moro E, Godeiro C, Reichmann H (2018) Medical and surgical management of advanced Parkinson's disease. Mov Disord 33:900-908

5. Chaudhuri KR, Odin P, Antonini A, Martinez-Martin P (2011) Parkinson's disease: the non-motor issues. Parkinsonism Relat Disord 17:717-723

6. Santos-García D, de la Fuente-Fernández R (2013) Impact of nonmotor symptoms on health-related and perceived quality of life in Parkinson's disease. J Neurol Sci 332(1-2):136-140

7. Lundqvist C, Beiske AG, Reiertsen O, Kristiansen IS (2014) Real life cost and quality of life associated with continuous intraduodenal levodopa infusion compared with oral treatment in Parkinson patients. J Neurol 261:2438-2445

8. Palhagen SE, Sydow O, Johansson A, Nyholm D, Holmberg B, Widner H, Dizdar N, Linder J, Hauge T, Jansson R, Bergmann L, Kjellander S, Marshall TS (2016) Levodopa-carbidopa intestinal gel (LCIG) treatment in routine care of patients with advanced Parkinson's disease: an open-label prospective observational study of effectiveness, tolerability and healthcare costs. Parkinsonism Relat Disord 29:17-23

9. Antonini A, Chaudhuri KR, Martinez-Martin P, Odin P (2010) Oral and infusion levodopa- based strategies for managing motor complications in patients with Parkinson's disease. CNS Drugs 24:119-129

10. Contin M, Martinelli P (2010) Pharmacokinetics of levodopa. J Neurol 257:253-261

11. Nutt JG (2008) Pharmacokinetics and pharmacodynamics of levodopa. Mov Disord 23:S580-S584

12. Chapuis S, Ouchchane L, Metz O, Gerbaud L, Durif F (2005) Impact of the motor complications of Parkinson's disease on the quality of life. Mov Disord 20:224-230

13. Antonini A, Stoessl AJ, Kleinman LS, Skalicky AM, Marshall TS, Sail KR, Onuk K, Odin PLA (2018) Developing consensus among movement disorder specialists on clinical indicators for identification and management of advanced Parkinson's disease: a multicountry Delphi-panel approach. Curr Med Res Opin 34:20632073. https://doi.org/10.1080/03007995.2018.1502165

14. Nyholm D, Askmark H, Gomes-Trolin C, Knutson T, Lennernäs H, Nyström C, Aquilonius SM (2003) Optimizing levodopa pharmacokinetics: intestinal infusion versus oral sustained- release tablets. Clin Neuropharmacol 26:156

15. Nyholm D, Odin P, Johansson A, Chatamra K, Locke C, Dutta S, Othman A (2013) Pharmacokinetics of levodopa, carbidopa, and 3O-methyldopa following 16-hour jejunal infusion of levodopacarbidopa intestinal gel in advanced Parkinson's disease patients, pharmacokinetics of levodopa, carbidopa, and 3-O-methyldopa following 16-hour Jejunal infusion of levodopa-carbidopa intestinal gel in advanced Parkinson's disease patients 15:316-323

16. Olanow CW, Kieburtz K, Odin P, Espay AJ, Standaert DG, Fernandez HH, Vanagunas A, Othman AA, Widnell KL, Robieson WZ, Pritchett Y, Chatamra K, Benesh J, Lenz RA, Antonini A (2014) Continuous intrajejunal infusion of levodopacarbidopa intestinal gel for patients with advanced Parkinson's disease: a randomised, controlled, double- blind, double- dummy study. Lancet Neurol 13:141-149

17. Fernandez HH, Standaert DG, Hauser RA, Lang AE, Fung VSC, Klostermann F, Lew MF, Odin P, Steiger M, Yakupov EZ, Chouinard S, Suchowersky O, Dubow J, Hall CM, Chatamra K, Robieson WZ, Benesh JA, Espay AJ (2015) Levodopa-carbidopa intestinal gel in advanced Parkinson's disease: final 12-month, open-label results. Mov Disord 30:500-509

18. Slevin JT, Fernandez HH, Zadikoff C, Hall C, Eaton S, Dubow J, Chatamra K, Benesh JA (2015) Long-term safety and maintenance of efficacy of levodopa-carbidopa intestinal gel: an open-label extension of the double-blind pivotal study in advanced Parkinson's disease patients. J Park Dis 5:165-174

19. Palhagen SE, Dizdar N, Hauge T, Holmberg B, Jansson R, Linder J, Nyholm D, Sydow O, Wainwright M, Widner H, Johansson A (2012) Interim analysis of long-term intraduodenal levodopa infusion in advanced Parkinson disease. Acta Neurol Scand 126:e29-e33

20. Antonini A, Odin P, Lopiano L, Tomantschger V, Pacchetti C, Pickut B, Gasser UE, Calandrella D, Mancini F, Zibetti M, Minafra B, Bertaina I, Deyn P, Cras C, Wolf E, Spielberger S, Poewe W (2013) Effect and safety of duodenal levodopa infusion in advanced Parkinson's disease: a retrospective multicenter outcome assessment in patient routine care. J Neural Transm 120: $1553-1558$

21. Bajenaru O, Ene A, Popescu BO, Szasz JA, Sabau M, Muresan DF, Perju-Dumbrava L, Popescu CD, Constantinescu A, Buraga I, Simu M (2016) The effect of levodopa-carbidopa intestinal gel infusion long-term therapy on motor complications in advanced Parkinson's disease: a multicenter Romanian experience. J Neural Transm 123: 407-414

22. Buongiorno M, Antonelli F, Camara A, Puente V, de FabreguesNebot O, Hernandez-Vara J, Calopa M, Pascual-Sedano B, Campolongo A, Valldeoriola F, Tolosa E, Kulisevsky J, Marti MJ (2015) Long-term response to continuous duodenal infusion of levodopa/carbidopa gel in patients with advanced Parkinson disease: the Barcelona registry. Parkinsonism Relat Disord 21: 871-876

23. Valldeoriola F, Grandas F, Santos-Garcia D, Regidor I, Catalan MJ, Arbelo JM, Puente V, Mir P, Parra JC (2016) Long-term effectiveness of levodopa-carbidopa intestinal gel in 177 Spanish patients with advanced Parkinson's disease. Neurodegener Dis Manag 6: 289-298

24. Zibetti M, Merola A, Artusi CA, Rizzi L, Angrisano S, Reggio D, De Angelis C, Rizzone M, Lopiano L (2014) Levodopa/carbidopa intestinal gel infusion in advanced Parkinson's disease: a 7-year experience. Eur J Neurol 21:312-318

25. Antonini A, Yegin A, Preda C, Bergmann L, Poewe W (2015) Global long- term study on motor and non- motor symptoms and safety of levodopa-carbidopa intestinal gel in routine care of advanced Parkinson's disease patients: 12-month interim outcomes. Parkinsonism Relat Disord 21:231-235

26. Antonini A, Poewe W, Chaudhuri KR, Jech R, Pickut B, Pirtošek Z, Szasz J, Valldeoriola F, Winkler C, Bergmann L, Yegin A, Onuk K, Barch D, Odin P, on behalf of the GLORIA study co-investigators (2017) Levodopa-carbidopa intestinal gel in advanced Parkinson's: final results of the GLORIA registry. Parkinsonism Relat Disord 45: $13-20$ 
27. Medical Dictionary for Regulatory Activities (MedDRA), Version 14.0 2011. Available at: https://www.who.int/medical_devices/ innovation/MedDRAintroguide_version14_0_March2011.pdf)

28. Tomlinson CL, Stowe R, Patel S, Rick C, Gray R, Clarke CE (2010) Systematic review of levodopa dose equivalency reporting in Parkinson's disease. Mov Disord 25(15):2649-2685

29. Hauser RA, Auinger P (2011) Determination of minimal clinically important change in early and advanced Parkinson's disease, movement disorders. J Mov Disord Soc 26:813

30. Antonini A, Robieson WZ, Bergmann L, Yegin A, Poewe W (2018) Age/disease duration influence on activities of daily living and quality of life after levodopa-carbidopa intestinal gel in Parkinson's disease. Neurodegener Dis Manag 8(3):161-170

31. Epstein M, Johnson DA, Hawes R, Schmulewitz N, Vanagunas AD, Gossen ER, Robieson WT, Eaton S, Dubow J, Chatamra K,
Benesh JA (2016) Long-term PEG-J tube safety in patients with advanced Parkinson's disease. Clin Transl Gastroenterol 7:e159. https://doi.org/10.1038/ctg.2016.19

32. Lang AE, Rodriguez RL, Boyd JT, Chouinard S, Zadikoff C, Espay AJ, Slevin JT, Fernandez HH, Lew MF, Stein DA, Odin P, Fung VSC, Klostermann F, Fasano A, Draganov PV, Schmulewitz N, Robieso WZ, Eaton S, Chatamra K, Benesh JA, Dubow J (2016) Integrated safety of levodopa-carbidopa intestinal gel from prospective clinical trials. Mov Disord 31:538-546

Publisher's note Springer Nature remains neutral with regard to jurisdictional claims in published maps and institutional affiliations. 